\title{
Descriptive Epidemiology of Gastrointestinal Cancers: Results from National Cancer Registry Programme, India
}

\author{
Shakuntala T S, Sathishkumar Kumar Krishnan, Priyanka Das, Kondalli \\ Lakshminarayana Sudarshan, Chitra M Kotian, Stephen Santhappan, Monesh \\ Baburao Vishwakarma, Sureshkumar N, Prashant Mathur*
}

\begin{abstract}
Purpose: This study aims to determine the incidence, histology, clinical extent of disease, and trends of gastrointestinal (GI) cancers in India. Methods: GI cancer cases diagnosed between 2012-2016 from 28 Population-Based Cancer Registries and 58 Hospital Based Cancer Registries under the National Cancer Registry Programme were included. Crude incidence rate and age-standardized incidence rates (AARs) were calculated. Joinpoint regression program, 4.0.1 was used for trend analysis for data from 1982 to 2016, and a P-value of $<<0.05$ was considered statistically significant. Results: GI cancers' occurrence was more common among men (60.5\%) than in women (39.5\%). The incidence of GI cancer was highest in India's northeast region, Aizawl district (AAR 126.9) among males, and in Papumpare district (AAR 75.9) among females. The commonest cancer among men was cancer of the esophagus (28.2\%), followed by stomach cancer $(21 \%)$ and rectum cancer (14.3\%). Among women, cancer of the esophagus $(25.7 \%)$, gallbladder $(23.8 \%)$, stomach (14.8\%), and rectum $(14.6 \%)$ were common. Adenocarcinoma $(57.83 \%)$ was the commonest type of GI tumors, followed by Squamous Cell neoplasms (25.99\%). Majority of the GI cancers presented at the locoregional stage, but cancer of the gall bladder and pancreas presented at advanced stages. A rising trend for cancers of the colon, rectum, liver, gall bladder, pancreas was seen, while a declining trend was observed for stomach and oesophageal cancer. Conclusion: Our study highlights an increasing magnitude of GI cancers across different regions of India. Cancer registries form an essential tool for surveillance of GI cancers thus guiding prevention, early detection, and control programs.
\end{abstract}

Keywords: Cancer registry- gastrointestinal cancers- incidence- trends- India

Asian Pac J Cancer Prev, 23 (2), 409-418

\section{Introduction}

Cancer is a significant public health problem worldwide as well as in India. The gastrointestinal (GI) cancers include cancer of the esophagus, stomach, small intestine, colon, rectum, anus \& anal canal, liver $\&$ intrahepatic bile ducts, gall bladder, and pancreas. Incidence of different GI cancer types varies between Asian and western countries. Colorectal and pancreatic cancers were highest in Europe and North America, while gastric, esophageal, and liver cancers were more common in Asia (Arnold et al., 2020). However, in India, cancer of the esophagus and stomach were common among males (Mathur et al., 2020). There is a high incidence of gallbladder cancer in the North and Northern-East parts of India in both sexes(ICMR-NCDIR, 2020). As per the Global Burden of Disease India study report (1990-2016), stomach cancer accounted for $9 \%$, colon and rectum $(5.8 \%)$ and esophageal (4.3\%), liver cancer (3.5\%) and gall bladder and biliary tract cancer $(3.1 \%)$ of the total cancer DALYs in India (India State-Level Disease Burden Initiative Cancer Collaborators, 2018).

For effective cancer prevention and control, data on the magnitude, patterns, and trends of GI cancers are essential. In India, since 1981, a systematic collection of data on cancer is being carried out under the National Cancer Registry Programme (NCRP) through the PopulationBased Cancer Registries (PBCRs) and the Hospital-Based Cancer Registries (HBCRs). The objective of this paper is to describe the incidence, clinical extent of GI cancers and their trends in India for the defined time periods.

\section{Materials and Methods}

We obtained gastrointestinal cancer cases diagnosed between 2012-2016 from 28 PBCRs and 58 HBCRs under the NCRP (ICMR-National Centre for Disease Informatics and Research, 2020). PBCRs provide information 
related to cancer statistics in a geographically defined population, while HBCRs record information related to cancer diagnosis and treatment of patients in a particular hospital (Jensen et al., 1991). In India, the process of cancer registration is active wherein trained registry staff collect data from different sources (hospitals, diagnostic laboratories, vital statistics departments) and enter in a standardized core form. Based on the location of the PBCRs, the country was categorized into 6 geographic zones: North, South, East, West, Central and Northeast. North zone consists of Delhi and Patiala registries. South zone includes registries of Hyderabad, Kollam, Thiruvananthapuram, Bangalore, and Chennai. East zone consists of Kolkata registry. The West zone comprises of Ahmedabad urban, Aurangabad, Osmanabad-Beed, Barshi rural, Mumbai, and Pune. The central registries were of Bhopal, Nagpur and Wardha. Manipur, Mizoram, Sikkim, Tripura, West Arunachal, Meghalaya, Nagaland, Pasighat, Cachar, Dibrugarh, and Kamrup urban registries comprise the north-east zone (Supplementary Figure 1).

International Classification of Diseases, Tenth Revision (ICD-10), and International Classification of Diseases for Oncology, 3rd Edition (ICD-O3) were used to code and classify data as per histological groups. All neoplasms with behavior code 3 as defined by ICD-O3 were included (Fritz et al., 2013). The cancer sites (as per ICD 10 codes) included in this study were esophagus (C15), stomach (C16), small intestine(C17), colon(C18), rectum $(\mathrm{C} 20)$ and anus and anal canal( $(\mathrm{C} 21)$, liver and intrahepatic bile ducts(C22), gall bladder \& other and unspecified parts of the biliary tract (C23-C24) and pancreas (C25).

The clinical extent of GI cancers before treatment was categorized into localized only, locoregional, distant metastasis, and unknown. Crude incidence rate and age-standardized incidence rates per 1,00,000 population were calculated using the world standard population (Segi,1960). Population denominators were derived from the Census of India, conducted by the Registrar General and Census Commissioner of India (Census of India, 2011). The census populations of 2001 and 2011 were used to calculate the interval population estimates for the years 2012 and 2016 by 5 -year age groups and sex (Takiar and Shobana, 2009). Cumulative risk (probability that an individual will be diagnosed with cancer between 0-74 year age group in the absence of any competing cause of death and assuming that the current trends prevail over the time period) was calculated for the GI cancers (Day, 1987). Age adjusted mortality rates (AAMR) were calculated for the age group between 0 to 74 years. Time trends in GI cancer incidence were calculated as annual percent change (APC) for the GI cancer cases diagnosed between 1982-2016 for select registries having long-duration data. Join point regression program, 4.0.1 (National Cancer Institute,) was used for trend analysis, and a P-value of $<0.5$ was considered statistically significant (Joinpoint regression program version 4.0.1, 2013).

NCRP has developed in-house software (PBCR and HBCR Data Management) for data capture, data quality checks, checks for duplicates, and mortality incidence matching. The list of errors was sent back to registries for clarifications and corrected at each level. Good-quality data indices of microscopic verification (MV \%) above $75 \%$, Death certificate only (DCO) $\%$ below $20 \%$, other and unspecified sites (O\&U\%) below 15\%, were calculated for each PBCR and accordingly classified (Bray and Parkin, 2009; Parkin and Bray, 2009; Mathur et al., 2020).

Ethics approval: The NCRP is a long-term registry which obtains Ethics committees approval from time to time. The last update was in 2020. Ethical approval by the ICMR-NCDIR - Institutional Ethics Committee (EC Ref: NCDIR/IEC/3019/2020) was obtained.

\section{Results}

The average number of GI cancer cases, crude incidence rate, cumulative risk, the median age at diagnosis, and mortality rate for 28 PBCRs under NCRP for males and females are given in Table 1. Among males, the life-time cumulative risk in India's northeast region ranged from 1 in 7 (Aizawl \& Papumpare district) to 1 in 49 (Manipur), while for the western registries it ranged from 1 in 30 (Mumbai) to 1 in 97 (Osmanabad \& Beed). The median age for diagnosis of GI cancers among males was 60 years. For females, the lifetime cumulative risk was highest in Papumpare district (1 in 11), followed by Aizawl district (1 in 14). The median age of diagnosis for GI cancers among females was 58 years.

Figure 1 illustrates the comparison of age-adjusted incidence rates (AARs) of GI cancers for all PBCRs. The AARs of GI cancer was highest among the NorthEastern registries. Among males, it was highest in Aizawl district (AAR 126.9), followed by Papumpare district (AAR 108.7) and East Khasi Hills district (AAR 102.4). In females, the age-adjusted incidence rate (AARs) of GI cancer was the highest in Papumpare district (AAR 75.9), followed by Aizawl district (AAR 58.8) and Kamrup Urban (AAR 56.2).

Among the specific GI cancer sites, the AAR for esophageal cancer incidence was highest in the East Khasi Hills district (Males: AAR 75.4, Females AAR: 33.6). The AAR for stomach cancer in males was highest in the Aizawl district (AAR 44.2) and Papumpare district (AAR 27.1) in females. Aizawl had the highest incidence for cancer of the colon (Males: AAR 7.2, Females: AAR 5.7), rectal cancer (Males: AAR 8.6, Females: AAR 5.7), and pancreatic cancer (Males: AAR 3.6, Females: AAR 4.1). The highest incidence of liver cancer was in the Papumpare district (Males: AAR 35.2, Females: AAR 14.4). Gall bladder (GB) cancer incidence among males was highest in Kamrup urban (AAR 7.9). Among females, the AAR for GB cancer was highest in Kamrup urban (AAR 16.2), followed by the Cachar district (AAR 11.9) and Delhi (AAR 11.6) (Supplementary Figure 2).

Figure 2 (a) depicts the trends over time of the GI cancer sites between 1982-2016 in select PBCRs. There was a rising trend of GI cancer in Bangalore (APC 0.83\% in males and $0.56 \%$ in females), Chennai (APC $0.56 \%$ in males, $0.73 \%$ in females), Delhi (APC $1.07 \%$ in males, $0.66 \%$ in females), Barshi rural registry (APC $1.15 \%$ in males) and among females in Bhopal registry (APC 


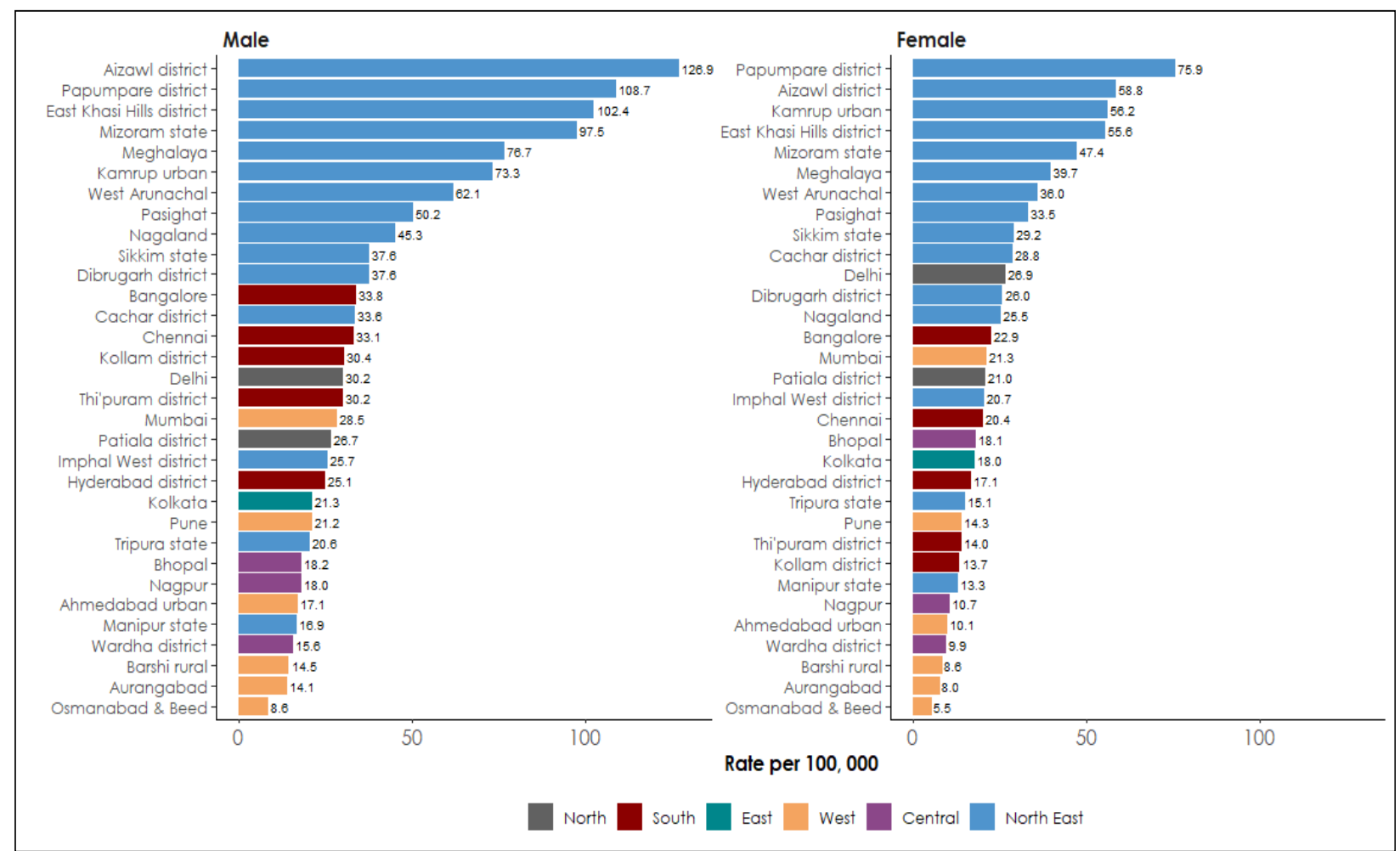

Figure 1. Comparison of Age-Adjusted Incidence Rates (AARs) of GI Cancers for 28 Population-Based Cancer Registries according to regions in India

$1.07 \%$ ). There was a declining trend of GI cancer in and Mumbai (APC $-0.45 \%$ in males, $-0.48 \%$ in females) and males in Bhopal (APC - $0.78 \%$ ).

Figure 2 (b) depicts the trends over time of the individual GI cancer sites between 1982-2016 for males. Esophageal cancer had a declining trend in Chennai,
Bhopal, Delhi, and Mumbai. Stomach cancer had a declining trend in Bangalore, Chennai, Bhopal, and Mumbai. In contrast, colon, rectum, and liver had rising trends in Bangalore, Chennai, Delhi, and Mumbai. Gall bladder cancer had rising trends in Delhi and Mumbai. There was a rising trend for pancreatic cancer in Chennai

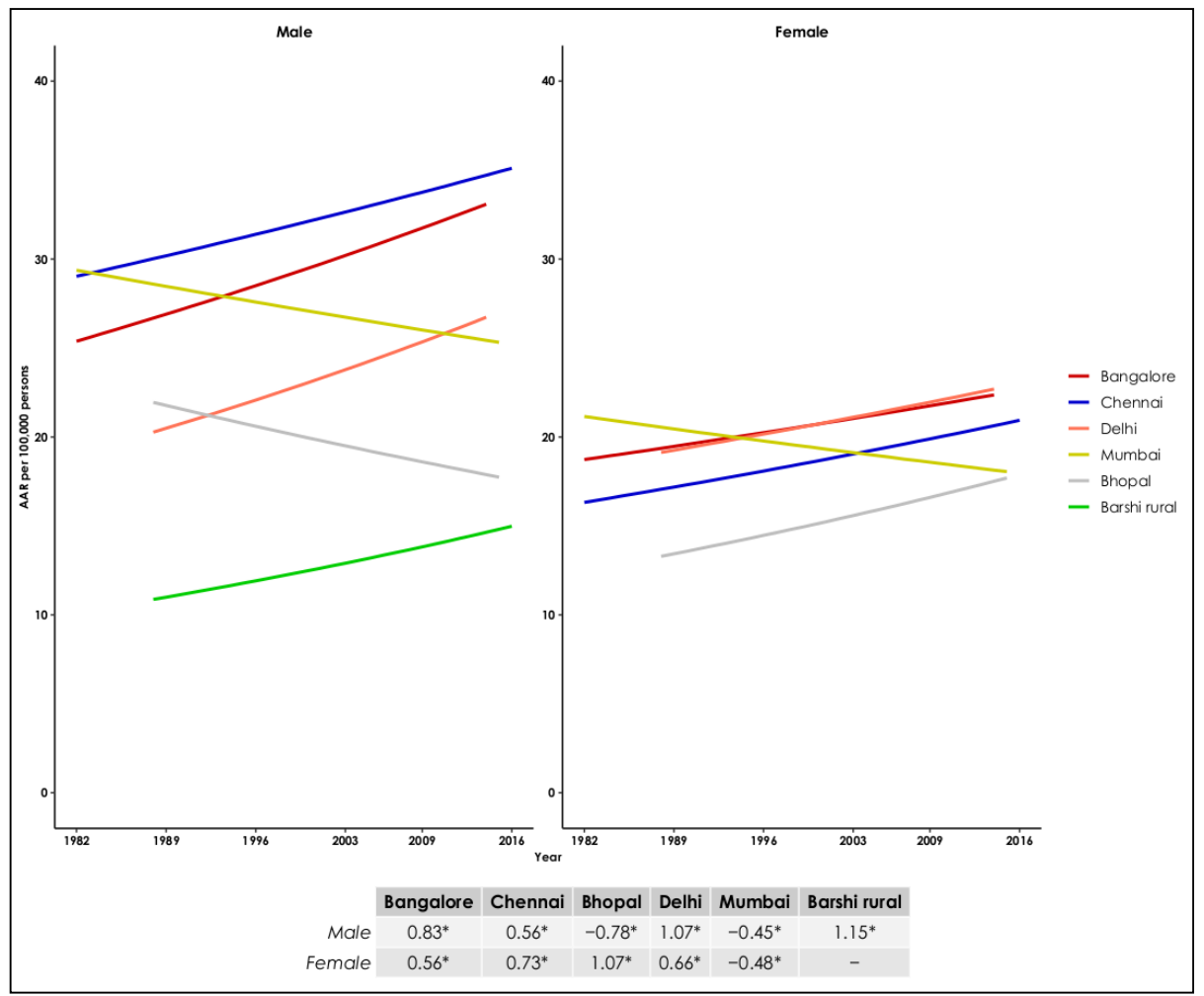

Figure 2 (a). Annual Percentage Change (APC) in Age-Adjusted Incidence Rates (AAR) of GI Cancer (1982-2016) in Males and Females. $\left.{ }^{*}\right)$ APC significantly different from zero; $\mathrm{P}<.05$. 


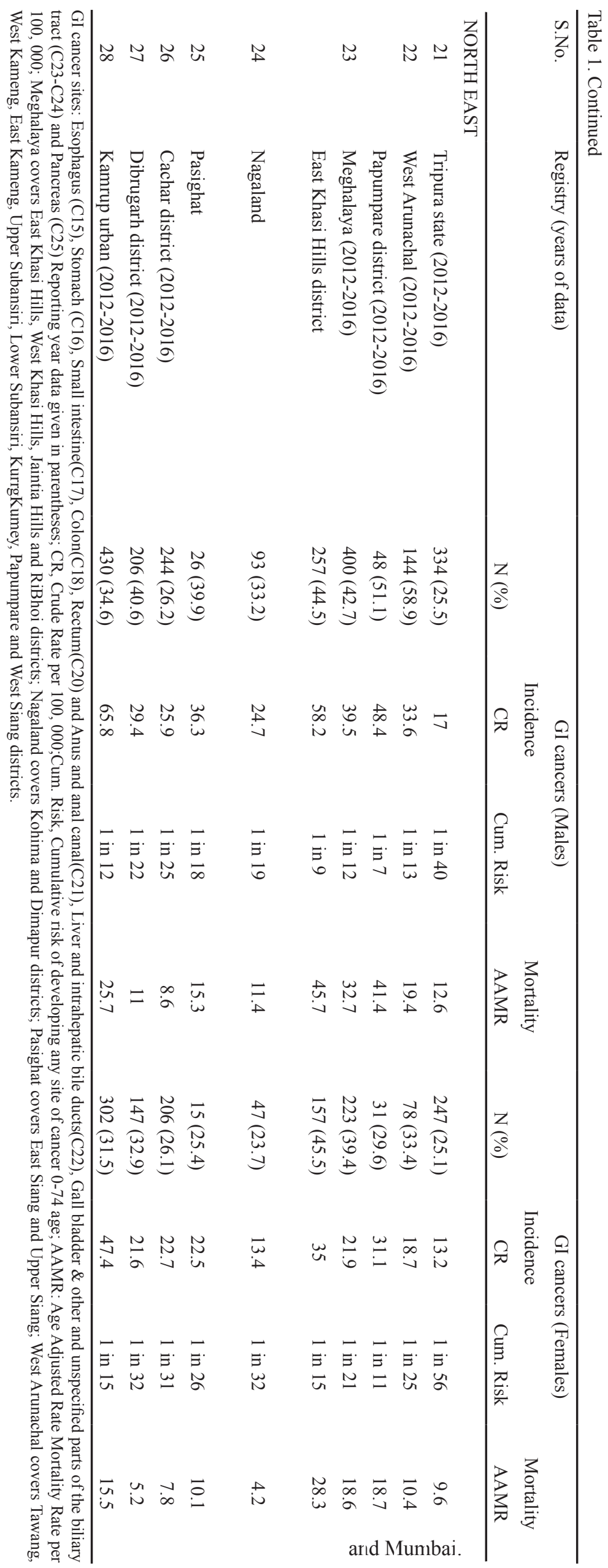




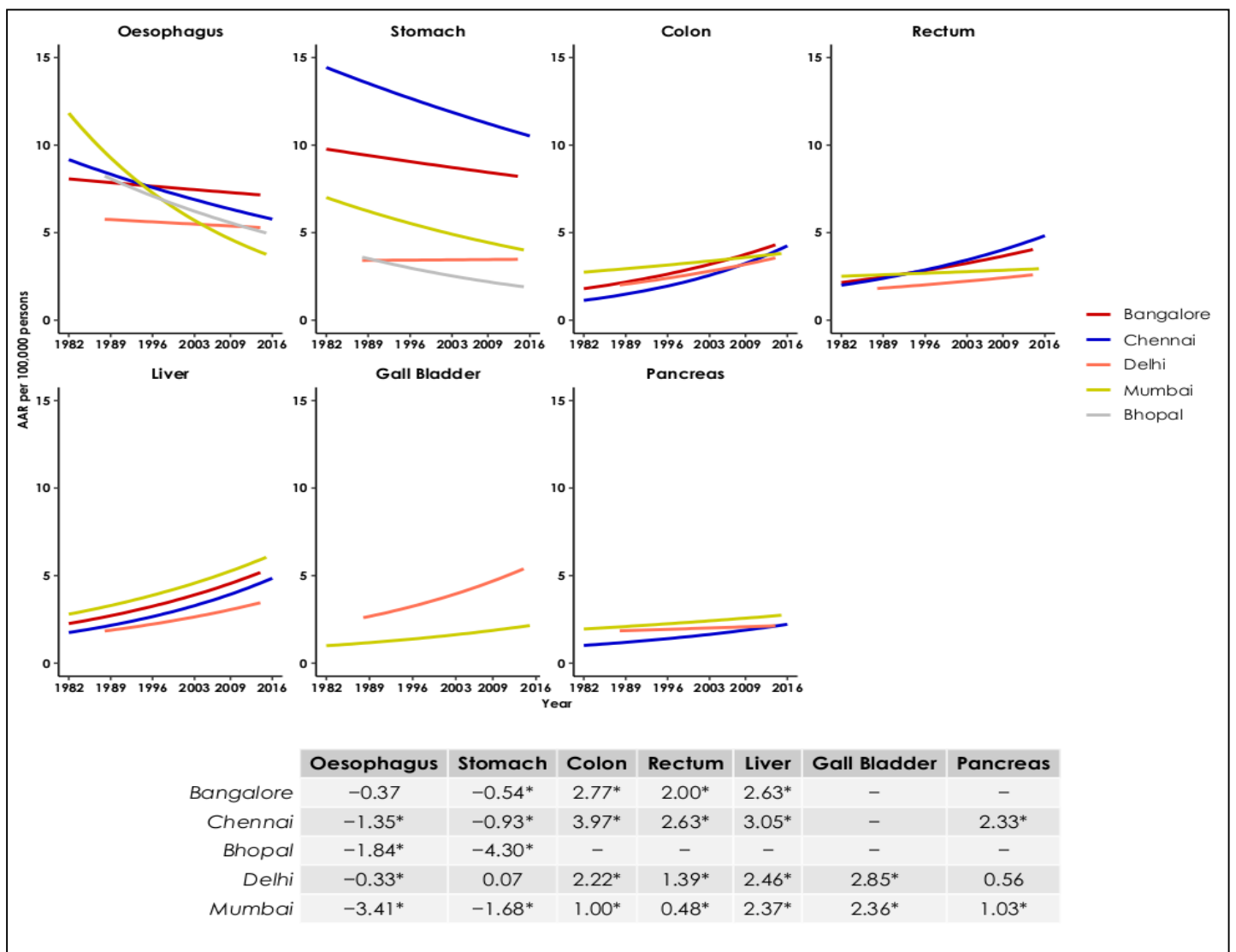

Figure 2 (b). Annual Percentage Change (APC) in Age-Adjusted Incidence Rates (AAR) of Individual GI Cancer Sites in Males (1982-2016)

Figure 2 (c) depicts the trends over time of the individual GI cancer sites between 1982-2016 for females. Esophageal cancer had a declining trend in Bangalore, Chennai, Delhi, and Mumbai. Stomach cancer had a declining trend in Chennai, Delhi, and Mumbai. In contrast, colon cancer had a rising trend in Chennai, Delhi, and Mumbai. Rectum cancer had rising trends in Bangalore, Chennai, and Delhi. Mumbai and Delhi had a rising trend for liver and gall bladder cancer. Pancreatic cancer had a rising trend among women in Mumbai.

Between 2012-2016, there were 54,229 GI cancer cases registered in 58 HBCRs. GI cancers' occurrence was more common among men $(60.5 \%)$ than in women
(39.5\%). Table 2 describes the proportion of cancer in different GI sites as registered in the HBCRs. The most prevalent cancer among men was cancer of the esophagus (28.2\%), followed by stomach cancer $(21 \%)$ and rectum cancer $(14.3 \%)$. Among women, the most common cancers were the cancer of the esophagus $(25.7 \%)$, gallbladder (23.8\%), stomach (14.8\%), and rectum (14.6\%).

Table 3 depicts the histological classification of GI cancers. Adenocarcinoma (57.8\%) was the most predominant type of GI tumor, followed by squamous cell neoplasms (26\%). Adenocarcinoma constituted $82.6 \%$ of rectal cancer, $79.9 \%$ in colon cancer, $76.3 \%$ of gall bladder cancer, $74.9 \%$ in stomach cancer, $49.3 \%$ in cancer of the

Table 2. Cumulative Frequency of Gastrointestinal Cancer in 58 HBCRs under NCRP

\begin{tabular}{lcccccc}
\hline GI Tumours & \multicolumn{2}{c}{ Males } & \multicolumn{2}{c}{ Females } & \multicolumn{3}{c}{ Total } \\
(ICD-10 codes) & $\mathrm{n}$ & $\%$ & $\mathrm{n}$ & $\%$ & $\mathrm{n}$ & $\%$ \\
\hline Esophagus (C15) & 9255 & 28.2 & 5490 & 25.7 & 14745 & 27.2 \\
Stomach (C16) & 6898 & 21 & 3158 & 14.8 & 10056 & 18.5 \\
Small Intestine (C17) & 327 & 1 & 196 & 0.9 & 523 & 1 \\
Colon (C18) & 2982 & 9.1 & 1943 & 9.1 & 4925 & 9.1 \\
Rectum (C19-20) & 4689 & 14.3 & 3134 & 14.6 & 7823 & 14.4 \\
Anus \& Anal Canal (C21) & 945 & 2.9 & 619 & 2.9 & 1564 & 2.9 \\
Liver (C22) & 2953 & 9 & 817 & 3.8 & 3770 & 7 \\
Gallbladder (C23-24) & 3297 & 10 & 5095 & 23.8 & 8392 & 15.5 \\
Pancreas (C25) & 1489 & 4.5 & 942 & 4.4 & 2431 & 4.5 \\
Total & 32835 & 100 & 21394 & 100 & 54229 & 100 \\
\hline
\end{tabular}




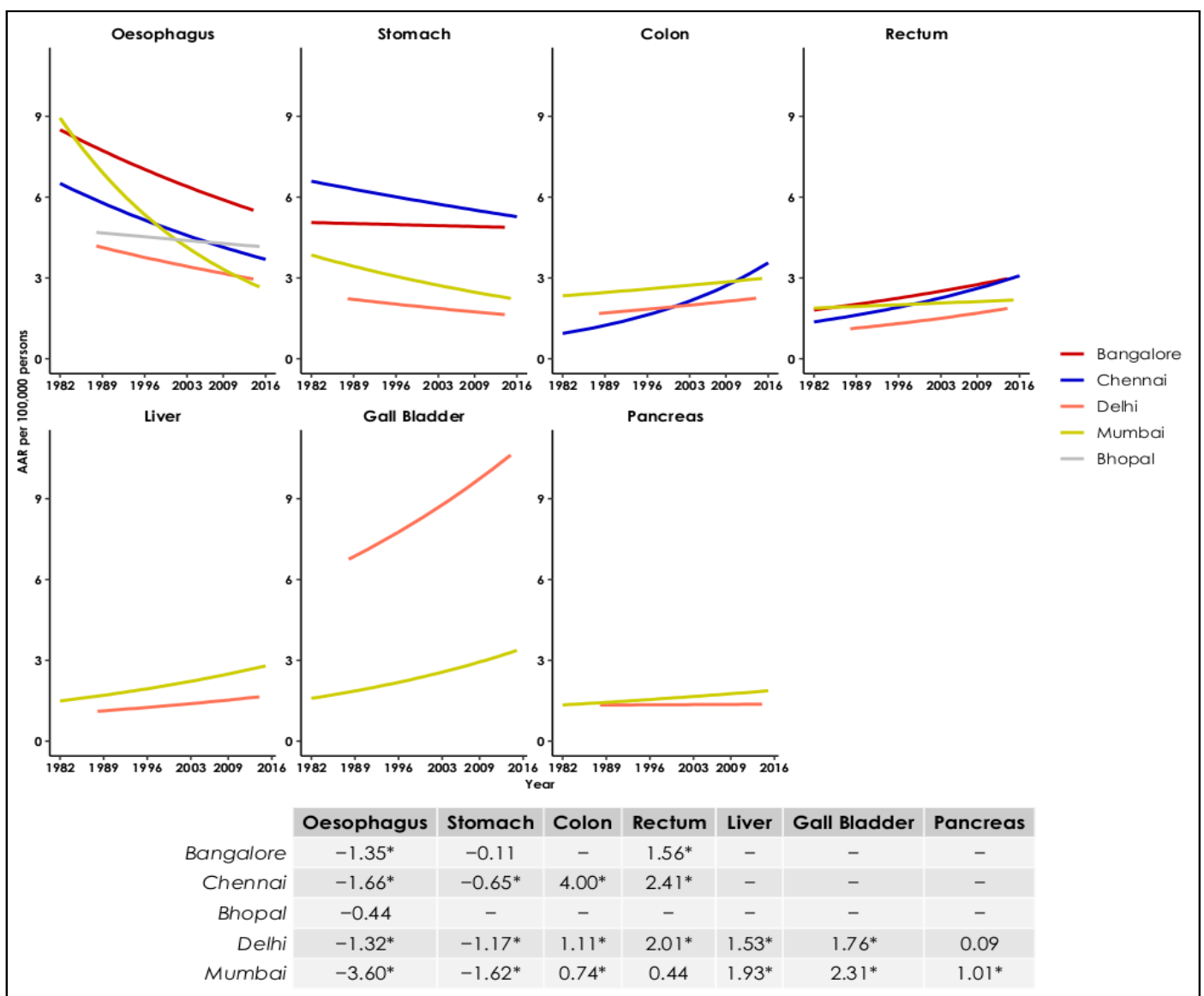

Figure 2 (c). Annual Percentage Change (APC) in Age-Adjusted Incidence Rates (AAR) of Individual GI Cancer Sites in Females (1982-2016

small intestine, $36.9 \%$ of anal cancer, and $10.6 \%$ of liver cancer. Squamous cell carcinoma, NOS was the leading

Table 3. Histological Classification of GI Cancers in 58 Hospital Based Cancer Registries under NCRP

\begin{tabular}{|c|c|c|}
\hline \multirow[t]{2}{*}{ Histological Groups } & \multicolumn{2}{|c|}{ Total } \\
\hline & $\mathrm{n}$ & $\%$ \\
\hline Adenocarcinoma & 31,359 & 57.8 \\
\hline Adenocarcinoma, NOS & 28,022 & \\
\hline Hepatocellular carcinoma, NOS (C22.0) & 1705 & \\
\hline Neuroendocrine carcinoma, NOS & 465 & \\
\hline Cholangiocarcinoma & 446 & \\
\hline Squamous cell neoplasms & 14,093 & 26 \\
\hline Squamous cell carcinoma, NOS & 13,216 & \\
\hline Squamous cell carcinoma, keratinizing, NOS & 403 & \\
\hline $\begin{array}{l}\text { Squamous cell carcinoma, large cell, } \\
\text { nonkeratinizing, NOS }\end{array}$ & 330 & \\
\hline Unspecified (PHM 8000-8002, 8004, 8005) & 2994 & 5.5 \\
\hline Cystic, mucinous and serous neoplasms & 2802 & 5.2 \\
\hline Signet ring cell carcinoma & 1618 & \\
\hline Mucinous adenocarcinoma & 640 & \\
\hline Mucin-producing adenocarcinoma & 512 & \\
\hline Epithelial neoplasms & 2001 & 3.7 \\
\hline Others & 718 & 1.3 \\
\hline Complex epithelial neoplasms & 204 & 0.4 \\
\hline Ductal and lobular neoplasms & 58 & 0.1 \\
\hline Total & 54,229 & 100 \\
\hline
\end{tabular}

histological subtype in esophageal cancer (85.8\%) and anal carcinomas $(44.5 \%) .45 .9 \%$ of liver cancers were hepatocellular carcinoma. Neuroendocrine carcinoma comprised $14.9 \%$ of cancer of small intestine. Signet ring cell carcinoma constituted $10.3 \%$ of stomach cancer and $4.1 \%$ of rectal cancer. Cholangiocarcinoma formed $6.4 \%$ of liver cancers and $2.4 \%$ of gall bladder cancers.

Figure 3 shows the relative proportion (\%) of the clinical extent of GI cancers at diagnosis among different GI sites for males and females. Locoregional (locally advanced) was the was the commonest presentation for the different types of GI cancers. Localized presentation was the second commonest presentation observed in all GI cancers except cancers of the stomach, gall bladder (males) and pancreas where distant metastasis was more common. Among the gall bladder cancers in females, distant metastasis (37.7\%) was the commonest presentation.

Data quality (28 PBCRs): The proportion of GI cancer cases with microscopic diagnosis was $82.1 \%$ and Death certificate only (DCO) was 6.6\%. The mortality/incidence ratio was $24.9 \%$ (Supplementary Table 1 ).

\section{Discussion}

This is the first comprehensive study from India on the major GI tract malignancies reported from in 28 PBCRs and 58 HBCRs under the National Cancer Registry Programme (NCRP). The North-Eastern registries had 
Males

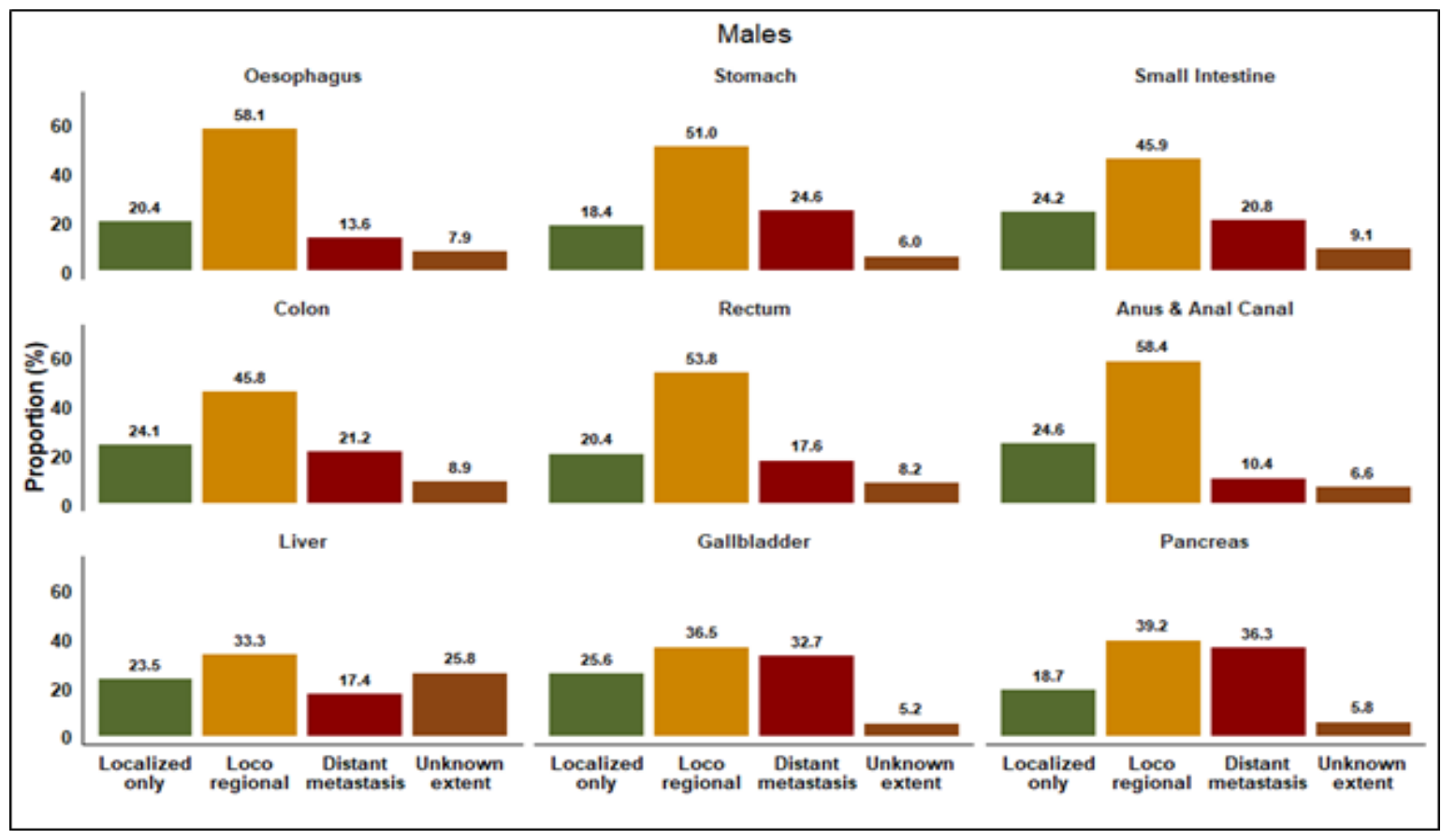

\section{Females}

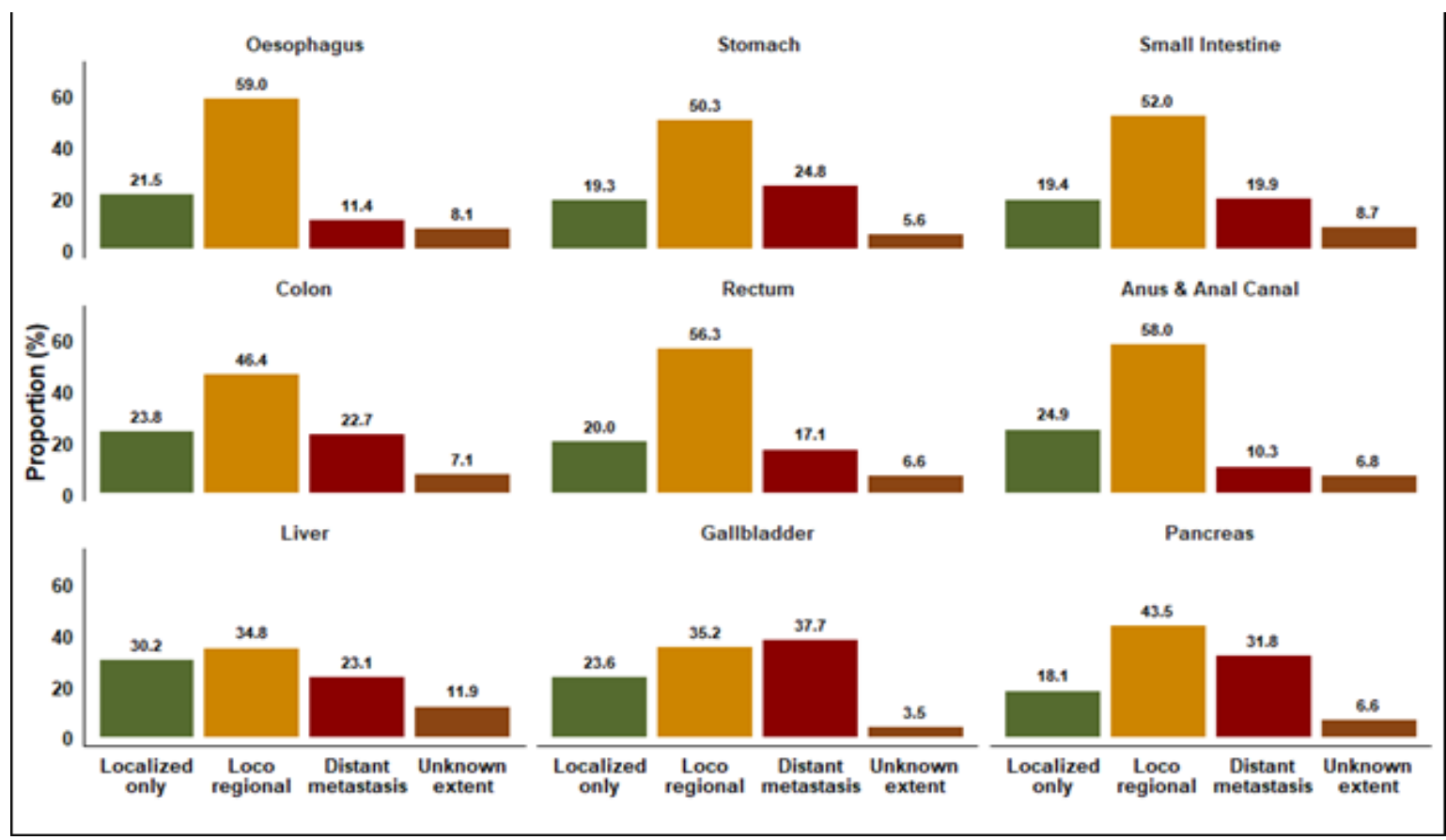

Figure 3. Relative Proportion (\%) of Clinical Extent of GI Cancer at Diagnosis in 58 Hospital Based Cancer Registries under the NCRP

higher age-adjusted incidence rates for GI cancers (16.9 to 126.9 in males and between 13.3 to 75.9 in females). Global comparison showed that the gall bladder cancer incidence among women in Kamrup urban (ASR 16.2) was second highest after Bio Bio in Chile (ASR 18.2). Gall bladder cancer incidence among men in Kamrup Urban (ASR 7.9) was the highest, followed by Bio Bio in Chile (ASR 7.2), Busan in the Republic of Korea (ASR 7.1) (Bray et al.,2017). The esophageal cancer incidence was highest in Cixian County in China (Males: ASR
132.4, Females: ASR 84.2,) followed by East Khasi Hills district (Males: ASR 75.4, Females ASR: 33.6) (Bray et al., 2017). The liver cancer incidence among females in Papumpare district (ASR 14.4) was second highest after Yanting County in China (ASR 25.1). Among males, the liver cancer incidence in the Papumpare district (ASR 35.2) was fourth highest after Qidong County, China (ASR 57.2), Khon Kaen, Thailand (ASR 40.7), Jejudo, Republic of Korea (ASR 39.2) (Bray et al., 2017).

Stomach cancers were second leading GI cancer in 
males and the third leading site among females. Based on our study findings, $21 \%$ of the males and $14.8 \%$ of the females developed stomach cancer in India. In Nepal, stomach cancer comprised $7.3 \%$ of all cancers among males and 4.4\% among females (Shrestha et al., 2020). The stomach cancer incidence in the Aizawl district (ASR 44.2) among males is fourth highest after China (Cixian County, ASR134.2), Japan (Yamagata, 62.2), and the Republic of Korea (Daejeon, ASR 60.2) (Bray et al., 2017). The age-standardized incidence rate for stomach cancer varied 11.5 times across Indian states as per the global burden of disease study report for India (India State-Level Disease Burden Initiative Cancer Collaborators, 2018).

Esophageal cancer had the commonest frequency of occurrence in males (28.2\%) and females (25.7\%). However, there was a declining trend for stomach and esophageal cancer seen in our data. Globally the trends in incidence of the stomach cancer have declined due to the improved sociodemographic index (GBD 2017 Stomach Cancer Collaborators, 2020). Similarly, for esophageal cancers, age-standardized incidence rates, mortality, and DALYs between 1990 and 2017 have decreased globally (Kamangar et al., 2020).

There was a rising trend for cancers of the colon, rectum, liver, gall bladder, and pancreas. Gall bladder cancer had rising trends in Delhi and Mumbai. Gall bladder cancers were more common among women and presented in the advanced stages, as observed in previous populatiuon-based studies (Lau et al., 2017; Akhtar-Danesh et al., 2020). Pancreatic cancer had a rising trend among women in Mumbai. There was an increasing trend for pancreatic cancer among men in Chennai and Mumbai.

The rising gastrointestinal cancers could be attributed to the changes in people's lifestyle behaviours (use of tobacco, alcohol and dietary habits). The improvements in the diagnosis and registrations also contribute to the increased incidence rates. Smoking is a known risk factor for GI cancers. Among dietary factors in India, spicy food, dried salted fish, smoked dried salted meat, and consumption of high-temperature foods are significant risk factors for gastric cancer (Dikshit et al., 2011). Mustard oil consumption is one of the risk factors for gall bladder cancers (Mhatre et al., 2020). Low intake of fruits and vegetables, tobacco in various forms such as smoking and smokeless tobacco, and alcohol consumption are common risk factors for gastric cancer and esophageal cancer (Samarasam, 2017; Gupta et al., 2018; Ghosh et al., 2020). Thus, GI cancers' primary prevention by dietary modifications and lifestyle changes plays a vital role.

Our cancer control programme does not target GI cancer screening or early detection, especially for the esophagus and stomach yet. South Asian countries with high stomach cancer incidence rates, such as Japan and South Korea, have population screening policies to detect stomach cancer (GBD 2017 Stomach Cancer Collaborators,2020). Gastrointestinal endoscopy helps in the diagnosis of precancerous conditions and early cancers. As there are disparities in GI cancer distribution, especially in the North-Eastern parts of the country

\section{Descriptive Epidemiology of Gastrointestinal Cancers in India}

and metropolitan cities, screening programs and early detection should be prioritized.

Since cancer is not a notifiable disease in India, cancer registration faces several challenges. The 28 population-based cancer registries in our study covers $10 \%$ of the population in India. However, the network of PBCRs and HBCRs under NCRP capture data from the different regional zones, and thus they provide a good representation of the incidence of GI cancers across the country.

In conclusion, our research shows a high magnitude of GI cancers (esophagus, stomach, gall bladder, rectum and pancreas) across different regions of India. Poor detection and inadequate screening strategies cause delayed diagnosis and thus leading to poorer outcome and survival. Cancer registries form an efficient tool for surveillance of GI cancers, thus guiding prevention, early detection and control programs.

\section{Author Contribution Statement}

Conception and design: Prashant Mathur, Shakuntala TS, Krishnan Sathishkumar. Data analysis and interpretation: Prashant Mathur, Shakuntala TS, Krishnan Sathishkumar, Priyanka Das, Kondalli Lakshminarayana Sudarshan, Chitra M Kotian, Stephen Santhappan, Monesh B Vishwakarma, N Sureshkumar. Manuscript writing: All authors. Final approval of manuscript: All authors. Accountable for all aspects of the work: All authors.

\section{Acknowledgments}

Authors acknowledge the contribution of 28 Population-Based Cancer Registries (PBCRs), 58 Hospital-Based Cancer Registries (HBCRs) under National Cancer Registry Programme.

\section{Funding}

Indian Council of Medical Research, Ministry of Health and Family Welfare, Government of India

\section{Ethical approval}

Ethical approval by the ICMR-NCDIR - Institutional Ethics Committee (EC Ref: NCDIR/IEC/3019/2020) was obtained.

\section{Availability of data}

The Report of National Cancer Registry Programme (ICMR-NCDIR) including data from 28 PBCRs and 58 HBCRs for the years 2012-2016 is available freely at https://www.ncdirindia.org/All Reports/Report 2020/ default.aspx. Further, data can be made available on specific request.

\section{Conflicts of interest}

There are no conflicts of interest.

\section{References}

Akhtar-Danesh N, Akhtar-Danseh GG, Seow H, Shakeel S, 
Finley C (2021). Treatment Modality and Trends in Survival for Gallbladder Cancer: a Population-Based Study. J Gastrointest Canc, 52, 256-62.

Arnold M, Abnet CC, Neale RE, et al (2020). Global Burden of 5 Major Types of Gastrointestinal Cancer. Gastroenterology, 159, 335-49.e15.

Bray F, Colombet M, Mery L, et al (2017). Cancer Incidence in Five Continents, Vol. XI (electronic version). Lyon: International Agency for Research on Cancer. Available from: https://ci5.iarc.fr.

Bray F, Parkin DM (2009). Evaluation of data quality in the cancer registry: Principles and methods. Part I: Comparability, validity and timeliness. Eur J Cancer, 45, 747-55.

Census of India. Office of the Registrar General and Census Commissioner. Socio Cultural Tables, C14, Population by Five Year Age Group, by Residence and Sex, 1991, 2001 and 2011. New Delhi, India. http://www.censusindia.gov.in

Day NE (1987). Cancer Incidence in Five Continents, Vol. V (IARC Scientific Publications No. 88), Cumulative rates and cumulative risk. International Agency for Research on Cancer, Lyon, pp 787-9.

Dikshit RP, Mathur G, Mhatre S, Yeole BB (2011). Epidemiological review of gastric cancer in India. Indian J Med Paediatr Oncol, 32, 3-11.

Fritz A, Percy C, Jack A, et al (2013). ICD-O International Classification of Diseases for Oncology, Third Edition, Geneva, pp 33-93.

GBD 2017 Stomach Cancer Collaborators (2020). The global, regional, and national burden of stomach cancer in 195 countries, 1990-2017: a systematic analysis for the Global Burden of Disease study 2017. Lancet Gastroenterol Hepatol, 5, 42-54.

Ghosh P, Mandal S, Mustafi SM, Murmu N (2020). Clinicopathological characteristics and incidence of gastric cancer in Eastern India: A Retrospective Study. $J$ Gastrointest Cancer, 52, 863-71.

Gupta S, Gupta R, Sinha D, et al (2018). Relationship between type of smokeless tobacco \& risk of cancer: A systematic review. Indian J Med Res, 148, 56-76.

ICMR-National Centre for Disease Informatics and Research (2020). Report of National Cancer Registry Programme (NCRP 2020). Bangalore, India.

India State-Level Disease Burden Initiative Cancer Collaborators (2018). The burden of cancers and their variations across the states of India: the Global Burden of Disease Study 1990-2016. Lancet Oncol, 19, 1289-306.

Jensen OM, Parkin DM, MacLennan R, et al (1991). Cancer Registration: Principles and Methods. Planning a cancer registry. IARC Scientific Publication No. 95, Lyon, France, $\mathrm{p} 23$.

Joinpoint regression program version 4.0.1 (2013). Statistical research and application branch, National Cancer Institute.

Kamangar F, Nasrollahzadeh D, Safiri S, et al (2020). The global, regional, and national burden of oesophageal cancer and its attributable risk factors in 195 countries and territories, 1990-2017: a systematic analysis for the Global Burden of Disease Study 2017. Lancet Gastroenterol Hepatol, 5, 582-97.

Lau CSM, Zywot A, Mahendraraj K, Chamberlain RS (2017). Gallbladder Carcinoma in the United States: A Population Based Clinical Outcomes Study Involving 22,343 Patients from the Surveillance, Epidemiology, and End Result Database (1973-2013). HPB Surg, 2017, 1-7.

Mathur P, Sathishkumar K, Chaturvedi M, et al (2020). Cancer Statistics, 2020: Report From National Cancer Registry Programme, India. JCO Glob Oncol, 6, 1063-75.
Mhatre S, Rajaraman P, Chatterjee N, et al (2020). Mustard oil consumption, cooking method, diet and gallbladder cancer risk in high- and low-risk regions of India. Int $J$ Cancer, 147, 1621-8.

Parkin DM, Bray F (2009). Evaluation of data quality in the cancer registry: Principles and methods Part II. Completeness. Eur J Cancer, 45, 756-64.

Samarasam I (2017). Esophageal cancer in India: Current status and future perspectives. Int $J$ Adv Med Heal Res, 4, 5-10.

Segi M (1960). Cancer mortality for selected sites in 24 countries (1950-1957). Department of Public Health, Tohoku University School of Medicine, Sendai, Japan.

Shrestha G, Neupane P, Lamichhane N, et al (2020). Cancer Incidence in Nepal: A Three-Year Trend Analysis 2013-2015. Asian Pac J Cancer Care, 5, 145-50.

Takiar R, Shobana B (2009). Cancer incidence rates and the problem of denominators - a new approach in Indian cancer registries. Asian Pac J Cancer Prev, 10, 123-6.

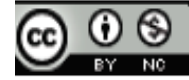

This work is licensed under a Creative Commons AttributionNon Commercial 4.0 International License. 